This item was submitted to Loughborough's Research Repository by the author.

Items in Figshare are protected by copyright, with all rights reserved, unless otherwise indicated.

\title{
Sleep loss and change detection in driving scenes
}

PLEASE CITE THE PUBLISHED VERSION

https://doi.org/10.1016/j.trf.2017.10.003

\section{PUBLISHER}

(C) Elsevier

\section{VERSION}

AM (Accepted Manuscript)

\section{PUBLISHER STATEMENT}

This work is made available according to the conditions of the Creative Commons Attribution-NonCommercialNoDerivatives 4.0 International (CC BY-NC-ND 4.0) licence. Full details of this licence are available at: https://creativecommons.org/licenses/by-nc-nd/4.0/

\section{LICENCE}

CC BY-NC-ND 4.0

\section{REPOSITORY RECORD}

Filtness, Ashleigh J., and Vanessa Beanland. 2019. "Sleep Loss and Change Detection in Driving Scenes". figshare. https://hdl.handle.net/2134/28450. 


\section{Sleep loss and change detection in driving scenes}

Filtness A.J ${ }^{1,2^{*}}$., \& Beanland $V^{3}$

1: Loughborough Design School, Loughborough University, LE11 3TU, UK

2: Queensland University of Technology, Centre for Accident Research and Road Safety Queensland (CARRS-Q), Kelvin Grove, QLD 4059, Australia

3: Centre for Human Factors and Sociotechnical Systems, University of the Sunshine Coast, Sippy Downs, QLD 4558, Australia.

*corresponding author: A.J.Filtness@lboro.ac.uk 


\section{Abstract}

Driver sleepiness is a significant road safety problem. Sleep-related crashes occur on both urban and rural roads, yet to date driver-sleepiness research has focused on understanding impairment in rural and motorway driving. The ability to detect changes is an attention and awareness skill vital for everyday safe driving. Previous research has demonstrated that person states, such as age or motivation, influence susceptibility to change blindness (i.e., failure or delay in detecting changes). The current work considers whether sleepiness increases the likelihood of change blindness within urban and rural driving contexts. Twenty fully-licenced drivers completed a change detection 'flicker' task twice in a counterbalanced design: once following a normal night of sleep (7-8h) and once following sleep restriction (5h). Change detection accuracy and response time were recorded while eye movements were continuously tracked. Accuracy was not significantly affected by sleep loss; however, following sleep loss there was some evidence of slowed change detection responses to urban images, but faster responses for rural images. Visual scanning across the images remained consistent between sleep conditions, resulting in no difference in the probability of fixating on the change target. Overall, theresults suggest that sleep loss has minimal impact on change detection accuracy and visual scanning for changes in driving scenes. However, a subtle difference in response time to change detection between urban and rural images indicates that change blindness may have implications for sleep-related crashes in more visually complex urban environments. Further research is needed to confirm this finding.

Keywords: driver sleepiness; sleepy drivers; driver fatigue; driver drowsiness; change detection; visual attention, Change blindness. 


\section{Highlights}

- Experimental investigation of sleep restriction impact on change blindness in driving scenes.

- Accuracy of change detection was maintained when sleepy.

- Visual search strategy was maintained when sleepy.

- When sleepy, there is some evidence that drivers detect changes more slowly in urban images, but faster in rural images.

- Change blindness may be an influencing factor in urban but not rural sleep-related crashes. 


\section{Introduction}

Driver sleepiness remains one of the leading contributors to road crashes, implicated in approximately 15-30\% of all crashes (Åkerstedt, 2000; Connor et al., 2002; Horne \& Reyner, 1995). Sleep-related crashes are particularly prevalent on high speed motorways and rural roads, where they are often high speed, serious injury, single vehicle, run-off-road events (Connor et al., 2002; Filtness, Armstrong, Watson, \& Smith, 2017; Philip et al., 2014).

Traditionally driver sleepiness research has focused on these environments (e.g. Anderson \& Horne, 2013; Anund, Kecklund, Vadeby, Hjälmdahl, \& Åkerstedt, 2008; Filtness, Reyner, \& Horne, 2012). However, sleep-related crashes are not confined to high speed roads: a recent study found that $41 \%$ of all police-reported sleep-related crashes occurred on low speed $(\leq 50 \mathrm{~km} / \mathrm{h}$ ) roads (Filtness et al., 2017). Drivers also self-report having sleepiness-related driving incidents on low speed urban roads (Armstrong, Filtness, Watling, Barraclough, \& Haworth, 2013). Urban roads present unique challenges in that they include a greater variety of road users (i.e., pedestrians, cyclists, motorised road users), have higher visual complexity, and the environment changes more frequently compared with rural areas. Understanding how sleep loss impairs driving ability in such complex situations, as well as lower complexity rural situations, is a first step to developing appropriate countermeasures to reduce crash risk.

One area that has received relatively little attention to date is the effect that sleepiness has on complex visual attention tasks such as change detection. The ability to detect changes is crucial for safe driving, and is positively correlated with safe decision making (Caird, Edwards, Creaser \& Horrey, 2005). Observers often fail to detect changes to visual scene, howerver, which is a psychological phenomenon known as change blindness (Rensink, O'Regan, \& Clark, 1997). This has implications for safe driving, especially the need to notice changes to the driving environment in order to respond appropriately. Change blindness in drivers has been observed using both static images of driving scenes (Beanland, Filtness, \& Jeans, 2017; Velichkovsky, Dornhoefer, Kopf, Helmert, \& Joos, 2002) and driving simulator paradigms (Charlton \& Starkey, 2013; Martens \& Fox, 2007). It is difficult to quantify the extent of crashes involving change blindness, or the failure to detect changes, but recent research suggests that the failure to detect vehicles or hazards, due to factors including 
change blindness, is a contributing factor in up to $10 \%$ of serious injury crashes (Beanland, Fitzharris, Young, \& Lenné, 2013).

\subsection{Change blindness}

The exact mechanism which underpins the identification of changes (and conversely the failure to achieve this which results in change blindness) is currently unknown. However, the ability to detect a change can be enhanced by both bottom-up mechanisms (e.g. external factors) or top-down mechanisms (e.g. internal person factors) and interactions between the two. External stimulus-related factors can include saliency and relevance of the change target. For example, changes to salient objects are detected faster (Theeuwes, 1994), and drivers are faster at detecting changes to task-relevant objects, such as road signs, compared with task-irrelevant changes such as to nearby buildings or walls (Galpin, Underwood, \& Crundall, 2009). The relevance of objects can also be influenced by instructions given to participants. Change detection is improved if participants are instructed to view the scene in a manner relevant to the changes being made. For example, instructing participants to make driving-related decisions about road images (e.g. is it safe to proceed across an intersection?) improves detection of vehicle change targets (Koustanaï, Van Elslande, \& Bastien, 2012). External factors may also interact, for example increasing the safety relevance of a change has a greater influence on accuracy and speed of change detection in visually cluttered urban driving scenes compared with rural driving scenes (Beanland et al., 2017). Internal person factors include: motivation, as observers who are motivated by money are more accurate at detecting changes (Sänger \& Wascher, 2011); and, age, as older adults are more susceptible to change blindness (Rizzo et al., 2009). Internal and external factors may also interact, for example, personal relevance (person factor) to the type of stimuli (external factor) improves change detection for that specific object. Insomnia patients are more likely to notice changes to sleep-related objects than non-sleeprelated objects in visual scenes (Marchetti, Biello, Broomfield, Macmahon, \& Espie, 2006), and experienced football players are more likely than novices to notice changes that are meaningful to game play (Werner \& Thies, 2000).

To be successful at change detection requires comparison of an image to a subsequent image, meaning the object of change must be tracked across the images. This is particularly 
relevant to driving as road environments are dynamic. In the real world many changes are accompanied by motion. Often it is noticing the motion itself which alerts the observer to the change occurring and directs attentional resources to track the object of interest (Rensink et al., 1997). However, change blindness is more likely to occur if there is a disruption to the visual scene; for example, if the object is obscured by something in the environment (such as the A-pillar of a car) or due to blinks or eye movements. Change blindness paradigms intentionally create visual interruptions to reproduce these situations (Velichkovsky et al., 2002). A common approach is the flicker paradigm (Rensink et al., 1997) where two images interspersed with a blank screen are repeatedly shown to an observer.

\subsection{Sleep loss and change blindness}

There has been almost no research examining the relationship between sleep and change blindness. However, sleep research does have an established history with the study of vigilance. Sustained vigilance is widely accepted to be one of the most sensitive mechanisms with which to study sleep-related impairment. The most commonly used tool is the psychomotor vigilance task (PVT). In this simple task participants must respond as quickly as possible to a visual stimulus (usually a digital timer counting up in milliseconds) presented at random intervals. PVT performance is sensitive to both time of day circadian variation (Cajochen, Khalsa, Wyatt, Czeisler, \& Dijk, 1999; Graw, Kräuchi, Knoblauch, Wirz-Justice, \& Cajochen, 2004; Van Dongen \& Dinges, 2000) and sleep loss (Anderson, Wales, \& Horne, 2010; Dinges et al., 1997; Van Dongen, Maislin, Mullington, \& Dinges, 2003).

When examining the detrimental effects of sleep loss on PVT performance, the most sensitive outcome measures are those which relate to the increase in the extremity of outliers: lapses (responses $>500 \mathrm{~ms}$ ), response time (RT) variability, and the slowest $10 \%$ of RTs (Basner \& Dinges, 2011). It is important to focus the RT analysis of any sleep loss investigation on the most poorly performed trials of a test battery because sleepy participants are susceptible to intermittent failures (or lapses) of performance, which can result from microsleeps (Dinges \& Kribbs, 1991). However, it has been demonstrated that not all PVT lapses result from microsleeps (eyes closed); some occur when the observer is distracted and has diverted their gaze, or when the observer has their eyes open and is looking at the task (Anderson et al., 2010). In particular, 95\% of shorter duration lapses 
(500-549ms) are likely to occur with eyes open (Anderson et al., 2010). It is possible that such "eyes open" lapses may also instate occurrences of change blindness, although there are key differences between the PVT and change blindness paradigms. PVT stimuli are always presented in the same spatial location, whereas change blindness paradigms (as in real driving) have spatial uncertainty. Spatial uncertainty in terms of the target object placement relative to the placement of a previous target and in relation to other irrelevant objects is known to influence performance during vigilance tasks (Helton, Weil, Middlemiss, \& Sawers, 2010; Mouloua \& Parasuraman, 1995). Consequently, eyes open lapses of sleepy observers during change blindness paradigms may result in greater impairment than the equivalent for PVT.

In addition to sustained vigilance decrements, several other research findings suggest that sleep loss may increase susceptibility to change blindness. Sleep loss increases double vision (Clark \& Warren, 1939) and exorphia, or divergence of the eyes outward (Horne, 1975) but decreases oculomotor function (De Gennaro, Ferrara, Urbani, \& Bertini, 2000; Fransson et al., 2008) which it turn impairs visual search performance (De Gennaro, Ferrara, Curcio, \& Bertini, 2001). Additionally, sleep-deprived observers are no longer able to employ optimal search strategies; instead a more exploratory approach is taken to environment searching (Glass et al., 2011). Furthermore, it has been demonstrated that both accuracy and RT for detecting threats in a simulated luggage search task is impaired following sleep loss (Basner et al., 2008). In the context of driving, these findings suggest that sleepy drivers may fail to focus their attention on the areas where safety-critical changes are most likely to occur. However, there is currently a lack of research investigating the visual scanning behaviour of sleepy drivers therefore it is unclear if sleep loss may result in an increasingly random scanning pattern as in Glass et al. (2011). Alternatively, sleepy drivers may have an increased reliance on pre-learned scan patterns coupled with impaired processing of what is looked at.

Sleepy individuals find it harder to maintain focus, compared with alert individuals. Comparing distractibility (i.e. diverting attention to a video playing in the room) during the traditional PVT vigilance task, sleep restriction to $5 \mathrm{~h}$ resulted in greater distraction, despite clear instruction to direct attention to the PVT (Anderson et al., 2010). The authors postulated that this susceptibility to distraction may have neuropsychological basis, as the 
prefrontal cortex is involved in suppression of distractions and is vulnerable to sleep loss (Harrison \& Horne, 1998; Nilsson et al., 2005). Furthermore, for prefrontal cortex controlled tasks, impairment of sleep deprived young adults is known to mirror that of healthy age related decline (Harrison, Horne, \& Rothwell, 2000). As aging is a person factor known to exacerbate change blindness (Rizzo et al., 2009), including detecting changes in road scenes (Batchelder, Rizzo, Vanderleest, \& Vecera, 2003; Caird et al.,2005), it is possible that sleep deprived young adults may experience impaired change detection following sleep loss in a manner which mirrors healthy aging.

\subsection{The current study}

In the current study we build on our previous work (Beanland et al., 2017) examining change blindness in the context of driving. Previous research has demonstrated, using different paradigms, that:

1. Change blindness is influenced by internal observer related factors and the observers relationship with the presented situation (e.g. observer age, motivation, or prior exposure to the situation); and,

2. Sleep loss impairs performance at both visual search performance and RT to sustained attention tasks, which require similar skills to change detection.

Therefore, it is hypothesised that when detecting changes between matched image pairs of driving scenes, a single night of reduced sleep will result in: (A) reduced change detection accuracy (increased change blindness); (B) increased RT to accurately identify changes; and (C) a less strategic (more random) visual scanning strategy, ultimately reducing the probability of fixating on the change target and increasing time to first fixation.

\section{Method}

\subsection{Participants}

Twenty-two participants were recruited but two participants dropped out after the first study session. Results are presented for 20 participants (14 female). All participants were 
aged $20-29$ years $(M=22.4, S D=2.4)$, provided informed consent and participated voluntarily in three sessions: one introductory session and two experimental sessions (see section 2.6 for session details). Participants were offered AUD\$50 compensation for their time, plus an additional allowance to cover travel expenses to attend the sleep restriction session. All participants had normal or corrected-to-normal visual acuity, as measured using a near vision chart, drove at least once a week, and had held a driving licence for at least 18 months.

Participants were pre-screened to ensure they met relevant inclusion criteria for participating in a sleep restriction study. Specifically, participants were required to be regular 7-8 hour/night sleepers who did not: take regular naps, suffer from extreme daytime sleepiness, or have any sleep disorders. Participants were excluded if they smoked, drank alcohol daily, and/or they consumed five or more high-caffeine drinks per day.

Ethical approval was given by the Australian National University Human Research Ethics Committee (protocol 2014/458).

\subsection{Design}

A repeated-measures design was used. All participants completed one introductory session and two experimental sessions. The introductory session was used to screen participants for suitability and to provide a sleep diary and movement monitor, which were used to record sleep prior to the experimental sessions. One experimental session was completed following a night of normal sleep (NS) and one after sleep restriction (SR) to five hours, which was achieved by instructing participants to delay bed-time by three hours on the night before the SR session. The order of experimental sessions (i.e., NS vs. SR first) was counterbalanced between participants.

\subsection{Apparatus}

Visual stimuli were presented on a 27" Apple iMac desktop computer. An Eyelink 1000 eyetracker, with a reported spatial accuracy within $0.25-0.5^{\circ}$, was used to monitor eye movements at a temporal frequency of $1000 \mathrm{~Hz}$. Head position was fixed using a chinrest with a viewing distance of $95 \mathrm{~cm}$, yielding a display area of $30.3^{\circ} \times 19.4^{\circ}$ visual angle. 
Stimulus presentation and data acquisition were controlled via SR Research Experiment Builder.

BodyMedia SenseWear Armbands were used to monitor participants' sleep and waking activity, to verify the sleep requirements were met during the three days preceding each testing session. SenseWear Armbands are wearable physiological monitoring devices that record several parameters; of particular relevance to the current study it records time spent lying down as well as sleep duration and efficiency.

\subsection{Stimuli}

Two matched sets of experimental stimuli (stimulus sets A \& B) were used, one for each change detection session. Each stimulus set included 80 image pairs depicting driving scenes: 20 urban image pairs which differed in a single detail (change-present pairs), 20 rural change-present pairs, 20 urban image pairs which were identical (change-absent pairs) and 20 rural change-absent pairs. In change-absent pairs the two images displayed were identical, whereas in change-present pairs the images were originally identical but one of the images was edited to add, remove or alter a single driving-relevant target. Drivingrelevant was defined as the occurrence of a change which would have the potential to influence driver behaviour. For example, the addition/removal of a vehicle as opposed to changing the colour of a vehicle. Urban image photographs were taken in Canberra (civic, inner north, Parliamentary Triangle), Australia, and rural images were taken on rural roads in surrounding regions.

Within both the urban and rural environments, five types of change targets were used. In the urban scenes the change targets were: cars, motorcycles, road signs, traffic lights or pedestrians, with four image pairs for each category. In the rural scenes the change targets were: cars, motorcycles, road signs, trees or animals, again with four image pairs for each category. Changes relating to cars, motorcycles, pedestrians, trees and animals involved the addition/removal of the change target. Changes related to road signs and traffic lights were alterations to the presented information (e.g. changing the traffic light colour from red to green, changing the speed limit posted on a sign by $10 \mathrm{~km} / \mathrm{h}$ ). 
All images subtended $23.0^{\circ} \times 17.5^{\circ}$ and were taken using a digital camera mounted on the dashboard of a station wagon. Images were photographed in areas local to the data collection (i.e., likely to be highly familiar to participants).

Image pairs were presented using a "flicker" sequence (see Figure 1), in which one image was presented for $500 \mathrm{~ms}$, followed by a 500 ms blank grey screen, followed by the second image for $500 \mathrm{~ms}$ and then another $500 \mathrm{~ms}$ blank. Images and blanks alternated until the participant responded, or for $30 \mathrm{~s}$, whichever occurred first. Participants were instructed to decide as quickly as possible whether a change occurred and then immediately press the space bar to register their decision. Once a participant pressed the space bar or after $30 \mathrm{~s}$ (whichever came first) they were then asked whether a change occurred (yes/no response). Participants were always required to explicitly report whether a change occurred. If they responded yes participants were then asked what the changed object was. Available response options were presented as a list on the screen and included "vehicle", "motorcycle", "bicycle", "person", "animal", "tree", "building", "sign", and "traffic light". Participants used a mouse to click their chosen option. Using a mouse allowed participants to maintain head position and therefore sustain accurate calibration of the eye tracker. 


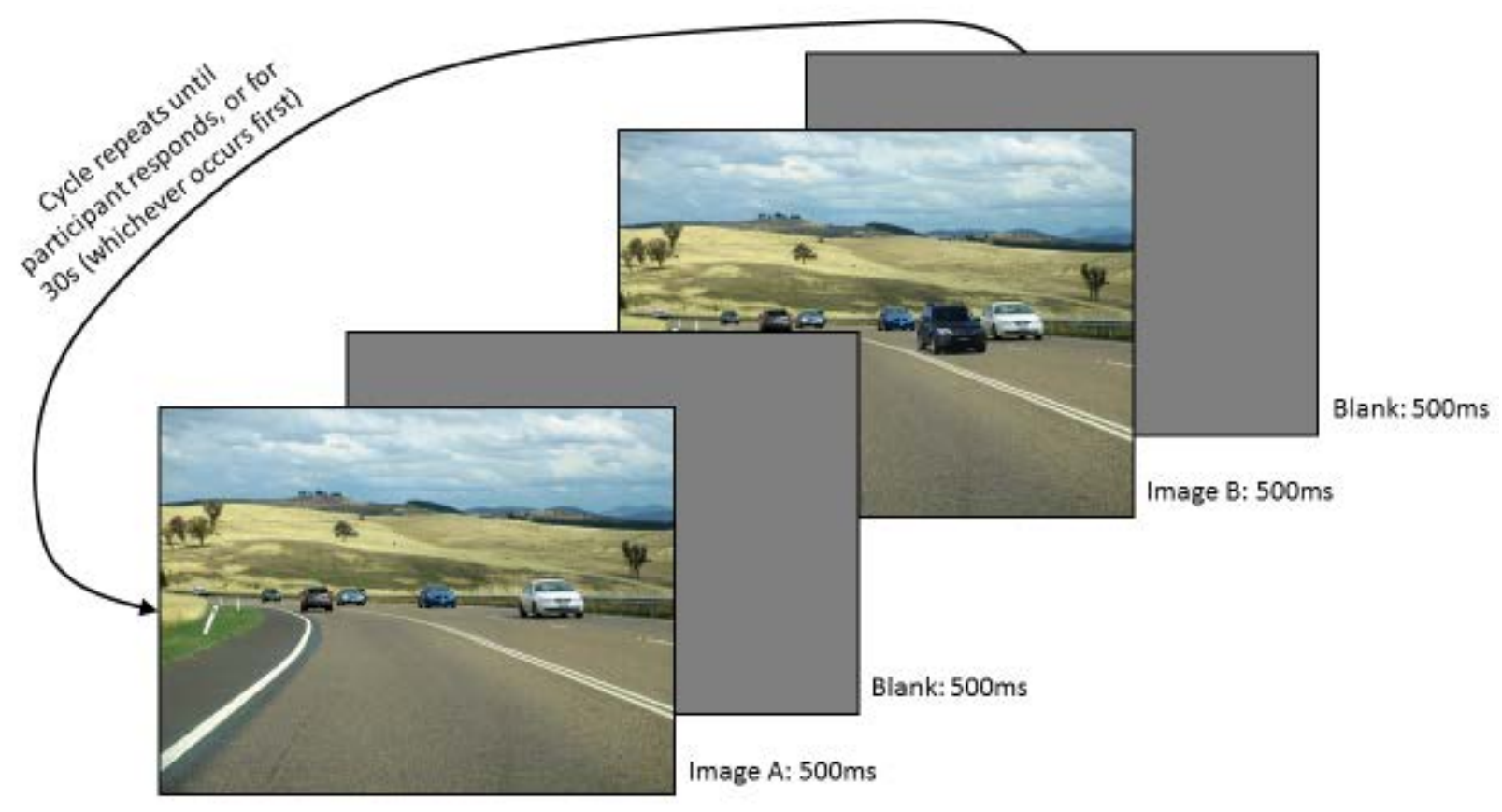

Fig. 1. Example trial sequence depicting a rural change-present trial in which the change target is a car. (For an example of an urban change-present trial sequence see Beanland et al., 2017.)

Change-present trials were considered "correct" if the observer correctly identified the changed object, but were considered "incorrect" if they reported no change or reported a change but then failed to select the correct target. Change-absent trials were considered "correct" if the observer reported no change, and were considered "incorrect" if they indicated that a change occurred.

Urban and rural trials were blocked (i.e. images of the same environment were shown in succession), with block order counterbalanced between participants, but trial order was randomised within each block. For both experimental sessions, the experimental task was preceded by 10 practice trials (with unique images presented in each practice block). The practice trials comprised five rural image pairs (three change-present, two change-absent) and five urban image pairs (three change-present, two change-absent).

The study design required repeated exposure to the same task, under both alert and sleepy conditions; however, the images used could not be identical to avoid learning bias. Therefore, matched stimulus sets were created using the stimuli from Beanland et al. (2017). Results for 100 change-present trials were analysed, comparing average RT and accuracy 
across all participants. Where two trials had the same scene context (i.e., urban or rural), change target, and similar difficulty (i.e., accuracy and RT), the image pair from one trial was assigned to stimulus set $A$ and the other image pair was assigned to stimulus set $B$. Some image pairs were not assigned to either stimulus set as they were outliers, in that change detection performance was unusually good (high accuracy, low RT) or poor (low accuracy, high RT). This resulted in 40 change-present image pairs for each stimulus set (80 total). Forty change-absent image pairs (20 urban, 20 rural) were also included in each stimulus set, with change-absent pairs similarly matched on RT and accuracy.

\subsection{Self-report measures}

Participants completed a demographics questionnaire, which included screening criteria and description of their usual driving exposure and behaviour. The Epworth Sleepiness Scale (ESS; Johns, 1991) was used to assess participants for excessive day time sleepiness (ESS>12).

For the three nights prior to each experimental study session participants were required to keep daily sleep diaries of their bed time, estimated sleep onset, night time wakings, and morning awakening and rising times. These self-report measures were considered alongside the objective SenseWear Armband recording of sleep.

Participants reported their subjective sleepiness on the Karolinska Sleepiness Scale (KSS; Åkerstedt \& Gillberg, 1990) at the start and end of each experimental session. The KSS measures state subjective sleepiness on a 9-point scale from 1 (extremely alert) to 9 (very sleepy, great effort to keep awake, fighting sleep).

\subsection{Procedure}

Participants attended three 30-minute sessions held on separate occasions at least three days apart. These comprised one introductory session followed by two experimental sessions. All sessions were on weekday afternoons, at either $1400 \mathrm{~h}$ or $1445 \mathrm{~h}$. Participants were tested individually in a quiet laboratory and completed all three sessions at the same time (i.e., a given participant would complete all sessions at $1400 \mathrm{~h}$, or all at $1445 \mathrm{~h}$ ). The 
presentation of stimulus sets was counterbalanced such that half the participants received stimulus set $A$ in the normal sleep session and B in the sleep restriction session, and viceversa.

In the introductory session participants provided written informed consent, completed the background questionnaires, and were given the SenseWear Armband and sleep diaries with instructions on how to use the armband and record their sleep.

In the experimental sessions, participants provided their sleep diary and armband to a research assistant, who checked the data to ensure compliance with the required hours of sleep. Participants were not allowed to proceed with the study if the experimental protocol had not been followed. The participant then completed the KSS to indicate their pre-task subjective sleepiness. Participants were seated in front of the computer with their head position stabilised using a chinrest. The eye-tracker was calibrated for each participant using a 16-point calibration grid and then validated to ensure the average gaze error was $<0.50^{\circ}$, which is within the manufacturer-specified margin of acceptable error. Each trial commenced with a drift check to ensure gaze calibration accuracy was maintained. Participants then completed the change detection task, which included 10 practice trials followed by 80 experimental trials, with a break halfway. The eye-tracker was recalibrated after the break to ensure accurate gaze tracking was maintained. Finally, after completion of the change detection task, there was a second administration of the KSS.

\subsection{Data analysis}

Statistical analyses were performed using SPSS v23. An alpha level of .05 was used to assess statistical significance.

Due to skewed data the reciprocal og variables were used for statistical analysis of: mean RT, slowest $10 \%$ of RT, and variability of RT (1/Standard deviation of RT). Mean and standard deviations of raw results are presented alongside the statistical analysis for reciprocal data.

Paired $t$-tests and repeated-measures analysis of variance (RM-ANOVA) were used to compare independent variables between NS and SR conditions. Paired $t$-tests results are reported with Cohen's $d$ as a measure of effect size calculated using the following formula 


$$
d=\frac{(\text { Mean } 1-\text { Mean 2) }}{((\mathrm{SD} 1+\mathrm{SD} 2) / 2)}
$$

Friedman tests were used for non-parametric data. Binary logistic Generalized Estimating Equations (GEE; Liang \& Zeger, 1986), specifying an exchangeable correlation matrix was used to analyse probability of fixating on target and probability of looked-but-failed-to-see errors (i.e. failing to detect the change, despite fixating the target). Binary logistic GEE in this case is used to assess whether the probability of a binary outcome differs according to the within-subjects variables of sleep condition.

It had been intended to analyse independent variables using RM-ANOVA with two withinsubjects factors: Sleep Condition (2 levels: NS, SR) and environment (2 levels: urban, rural). Change targets in rural and urban images were matched in terms of static/dynamic characteristics to allow this. However, participants demonstrated superior change detection for identifying person and traffic light changes (urban) compared with animal and tree changes (rural); therefore results from the two environmental conditions were analysed separately. Three analyses were conducted for each dependent variable: urban change detection; rural change detection; and urban/rural comparison. The urban analysis used results from all urban trials. The rural analysis used results from all rural trials. The urban/rural comparison considered results only from those trials with common change target objects (car, motorcycle, road sign).

\section{Results}

\subsection{Participants}

Participants were frequent drivers $(M=8.0$ hours/week, $S D=8.9$, range: $1-42)$, covering a mean of $263 \mathrm{~km} /$ week ( $S D=258$, range: 30-1000). No participants suffered from excessive daytime sleepiness, defined as ESS scores above $12(M=4.3, S D=2.5$, range: $0-11)$.

The order of sleep conditions (NS and SR) was fully counterbalanced; however, due to dropouts, there was some inconsistency in the counterbalancing of image sets used. For the NS session, 7 participants viewed image set $A$, whereas 13 participants viewed image set $B$. 


\subsection{Indicators of sleepiness}

Paired $t$-tests were used to compare the sleep duration and ocular indicators of sleepiness between NS and SR conditions. Participants slept an average of 494 minutes (SE $=8$ ) prior to the NS condition. Sleep was significantly restricted to an average of 303 minutes $(S E=7)$ during the SR condition, $t(19)=15.660, p<.001, d=5.59$.

Ocular indicators of sleepiness were calculated based on eye movements during correct change-absent trials only, as the presence or detection of a target would alter scanning and fixation patterns. The sleepiness related ocular indicators of interest were pupil size and "visual tunnelling". Tunnelling of vision is apparent if a greater proportion of gaze time occurs within a smaller area. There was no significant effect of sleep condition on any of the ocular indicators of sleepiness (see Table 1).

\section{Table 1}

Ocular indicators of sleepiness, comparing sleep conditions. Values represent mean from all correct change-absent trials, with standard error shown in parentheses.

\begin{tabular}{|c|c|c|c|}
\hline Measure & $\begin{array}{l}\text { Normal } \\
\text { Sleep }\end{array}$ & $\begin{array}{c}\text { Sleep } \\
\text { Restriction }\end{array}$ & Comparison \\
\hline Average pupil size & $1145(72)$ & $1130(64)$ & $\begin{array}{c}t(19)=0.397, p=.696 \\
d=0.05\end{array}$ \\
\hline $\begin{array}{l}50^{\text {th }} \text { percentile deviation from } \\
\text { average fixed location (pixels) }\end{array}$ & $204.3(5.3)$ & $205.5(4.5)$ & $\begin{array}{c}t(19)=0.411, p=.686, \\
d=0.06\end{array}$ \\
\hline $\begin{array}{l}70^{\text {th }} \text { percentile deviation from } \\
\text { average fixed location (pixels) }\end{array}$ & $291.1(7.6)$ & $292.6(7.2)$ & $\begin{array}{c}t(19)=0.423, p=.677, \\
d=0.05\end{array}$ \\
\hline $\begin{array}{l}90^{\text {th }} \text { percentile deviation from } \\
\text { average fixed location (pixels) }\end{array}$ & $462.4(10.4)$ & $462.0(9.0)$ & $\begin{array}{c}t(19)=0.071, p=.944, \\
d=0.01\end{array}$ \\
\hline
\end{tabular}

RM-ANOVA was used to compare subjective sleepiness with two within-subjects factors: Sleep condition (NS, SR) and time (before vs. after testing). Participants felt significantly sleepier following SR compared with NS, $F(1,19)=101.03, p<.001, \eta_{p}^{2}=.84$. Subjective sleepiness did not alter during testing, $F(1,19)=1.00, p=.330, \eta_{\mathrm{p}}^{2}=.05$ (see Table 2 ).

\section{Table 2}

Mean (and standard error) subjective sleepiness before and after testing for the two experimental sessions.

\begin{tabular}{lcc}
\hline & Normal Sleep & Sleep Restriction \\
\hline KSS before task & $3.5(0.3)$ & $6.4(0.3)$ \\
\hline
\end{tabular}




\subsection{Change detection accuracy}

Accuracy for change-absent trials was at ceiling following both NS (99.3\% rural images, $99.5 \%$ urban images) and SR (99.3\% rural images, 98.5\% urban images), so was not included in any statistical analysis. Accuracy for change-present trials was compared between sleep conditions using Friedman tests. Accuracy did not significantly differ between sleep conditions for either urban scenes (NS: $M=82.8 \%, S E=1.4 \%$; $S R: M=84.8 \%, S E=1.7 \%$ ), $\chi^{2}(1)=1.14, p=.285$, or rural scenes (NS: $M=78.8 \%, S E=1.7 \%$; SR: $M=76.0 \%, S E=2.0 \%$ ), $\chi^{2}(1)=0.60, p=.439$.

\subsection{Change detection RT}

RT variables were analysed for correct change-present trials only. Paired $t$-tests were used to compare between the NS and SR conditions for urban and rural images separately. Subsequently, RM-ANOVA analysis was conducted with two within-subjects factors: Sleep condition (NS, SR) and environment (urban, rural).

For the initial analyses considering urban and rural scenes separately, and including all correct trials for a particular driving environment regardless of change target type, there was no significant effect of sleep condition on any of the RT measures, as shown in Table 3. 


\section{Table 3}

Effects of sleep restriction on change detection RT, averaged across all target types, for separately analysed urban and rural scenes (no comparison between environments). Standard error shown in parentheses.

\begin{tabular}{|c|c|c|c|}
\hline & $\begin{array}{l}\text { Normal } \\
\text { Sleep }\end{array}$ & $\begin{array}{c}\text { Sleep } \\
\text { Restriction }\end{array}$ & Comparison \\
\hline \multicolumn{4}{|l|}{ Urban scenes } \\
\hline Mean RT (ms) & $5546(145)$ & $5765(203)$ & $\begin{array}{c}t(19)=1.273, p=.218 \\
d=0.27\end{array}$ \\
\hline Mean slowest $10 \%$ of RT (ms) & $7622(336)$ & 7905 (401) & $\begin{array}{c}t(19)=0.735, p=.472 \\
d=0.16\end{array}$ \\
\hline Variability of RT (ms) & $1032(108)$ & $1085(110)$ & $\begin{array}{c}t(19)=1.103, p=.284 \\
d=0.24\end{array}$ \\
\hline \multicolumn{4}{|l|}{ Rural scenes } \\
\hline Mean RT (ms) & $5560(196)$ & $5679(243)$ & $\begin{array}{c}t(19)=0.358, p=.724 \\
d=0.07\end{array}$ \\
\hline Mean slowest $10 \%$ of RT (ms) & $7974(588)$ & $8039(588)$ & $\begin{array}{c}t(19)=0.016, p=.987, \\
d=0.03\end{array}$ \\
\hline Variability of RT (ms) & $1208(180)$ & 1168 (159) & $\begin{array}{c}t(19)=0.317, p=.755 \\
d=0.07\end{array}$ \\
\hline
\end{tabular}

\subsubsection{Urban vs Rural comparison}

For the analysis directly comparing change detection performance in urban and rural scenes, only change-present trials with changes involving cars, motorcycles and road signs were included (as other targets were not consistent between environments). As shown in Table 4, there was a significant effect of environment on mean RT, with RT to rural images being faster than to urban images. There was also a significant interaction between sleep condition and environment for slowest $10 \%$ of RT and RT variability. As shown in Figure 3, sleep restriction slowed the slowest RTs to urban images, but increased it for rural images. Consistent with this, sleep restriction increased the variability of RT to urban images, while it decreased variability of RT to rural images. 
Table 4

Effects of sleep restriction and environment on change detection RT in change-present rural and urban images (car, motorcycle, road sign change targets only), standard error shown in parentheses.

\begin{tabular}{|c|c|c|c|c|c|c|c|}
\hline \multirow[b]{2}{*}{ Measure } & \multicolumn{2}{|c|}{ Normal Sleep } & \multicolumn{2}{|c|}{ Sleep Restriction } & \multicolumn{3}{|c|}{ Comparison } \\
\hline & Urban & Rural & Urban & Rural & Sleep & Environment & Interaction \\
\hline Mean RT (ms) & $5500(157)$ & $5424(173)$ & $5771(204)$ & $5497(210)$ & $\begin{aligned} & F=0.707, p \\
&=.411, \eta_{\mathrm{p}}^{2}=.04\end{aligned}$ & $\begin{array}{c}F=4.675, p \\
=.044, \eta_{\mathrm{p}}^{2}=.20\end{array}$ & $\begin{aligned} & F=1.616, p \\
= & .219, \eta_{\mathrm{p}}^{2}=.08\end{aligned}$ \\
\hline Mean slowest $10 \%$ of RT (ms) & $7438(404)$ & 7366 (401) & 7807 (389) & $7023(386)$ & $\begin{array}{c}F=0.00, p= \\
1.00, \eta_{\mathrm{p}}^{2}=.00\end{array}$ & $\begin{aligned} & F=3.212, p \\
= & .089, \eta_{\mathrm{p}}^{2}=.15\end{aligned}$ & $\begin{array}{c}F=6.876, p \\
=.017, \eta_{\mathrm{p}}^{2}=.27\end{array}$ \\
\hline Variability of RT (ms) & $1003(148)$ & $1350(274)$ & $1123(138)$ & $962(147)$ & $\begin{aligned} & F=0.00, p \\
&=.993, \eta_{\mathrm{p}}^{2}=.00\end{aligned}$ & $\begin{array}{c}F=0.078, p \\
=.784, \eta_{\mathrm{p}}^{2}=.00\end{array}$ & $\begin{array}{c}F=17.942, p \\
<.001, \eta_{\mathrm{p}}^{2}=.49\end{array}$ \\
\hline
\end{tabular}

Note. Degrees of freedom were $(1,19)$ for all $F$ tests. Statistically significant results are highlighted in bold. 

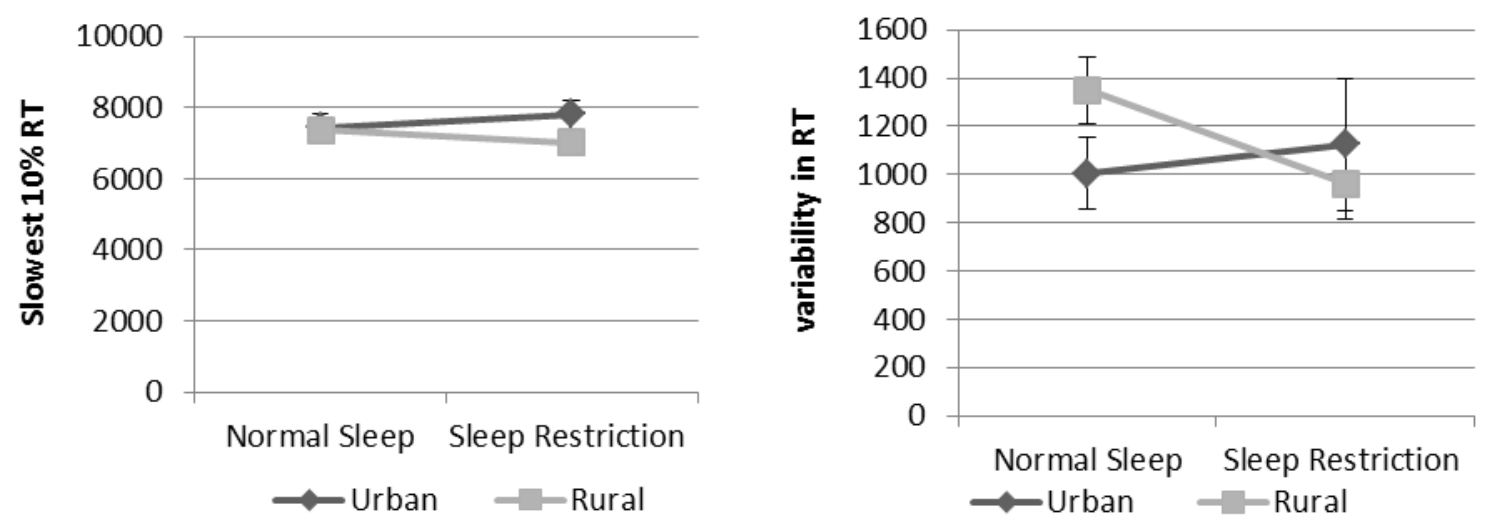

Fig. 3. Slowest $10 \%$ of RT for (left) and variability in RT (right) for correct change identification, by sleep condition and environment. Error bars represent standard error.

\subsection{Eye movements}

Eye moments were analysed with respect to fixations on the change target, and fixations in semantic interest areas (e.g., road vs. off-road areas) within the scene. Analyses relating to target fixations necessarily included change-present trials only. Four aspects of target fixation were selected for analysis: probability of fixating target; probability of looked-butfailed-to-see errors (i.e. failing to detect the change, despite fixating it); time to first fixation on the target; and total dwell time on target. As with change detection performance, three sets of analysis were undertaken: urban; rural; and urban/rural comparison. Analyses relating to semantic interest areas included change-absent trials only, as the presence of the target in change-present trials would have influenced natural eye movements.

\subsubsection{Probability of fixating target}

In order to represent implicit capture of attention, probability of fixation was analysed for all change-present trials regardless of accuracy. Binary logistic GEE compared whether sleep condition affected the probability of fixating the target.

Within urban scenes, there was no significant difference in probability of fixating the target when comparing SR $(M=61 \%, S E=5 \%)$ and NS $(M=62 \%, S E=4 \%)$ conditions, $\chi^{2}(1)=0.211$, $p=.646, B=-0.05, S E=0.11, \mathrm{OR}=0.95,95 \% \mathrm{Cl}$ OR $[0.76,1.19]$. 
Within rural scenes, there was also no significant difference in probability of fixating the target when comparing SR $(M=49 \%, S E=4 \%)$ and NS $(M=53 \%, S E=3 \%)$ conditions, $\chi^{2}(1)=$ 1.397, $p=.237, B=-0.17, S E=0.14, \mathrm{OR}=0.84,95 \% \mathrm{Cl}$ OR $[0.64,1.12]$.

In the analysis comparing probability of target fixation between urban and rural scenes (including car, motorcycle, and sign targets only) there was no significant effect of sleep condition, $\chi^{2}(1)=0.138, p=.668, B=-0.07, S E=0.16, \mathrm{OR}=0.93,95 \% \mathrm{Cl}$ OR $[0.68,1.28]$, and no significant effect of environment, $\chi^{2}(1)=1.422, p=.233, B=0.27, S E=0.22, \mathrm{OR}=1.30$, $95 \% \mathrm{Cl}$ OR $[0.84,2.02]$. Finally, there was no significant interaction between sleep condition and environment $\chi^{2}(1)=0.098, p=.754, B=0.07, S E=0.22, \mathrm{OR}=1.07,95 \% \mathrm{Cl}$ OR [0.70, 1.64].

\subsubsection{Probability of looked-but-failed-to-see errors}

Within urban scenes, $7.5 \%$ of all trials involved looked-but-failed-to-see errors. Binary logistic GEE indicated probability of looked-but-failed-to-see errors was not significantly different between SR $(M=6 \%, S E=2 \%)$ and NS $(M=8 \%, S E=1 \%)$ conditions, $\chi^{2}(1)=0.659$, $p=.417, B=-0.28, S E=0.35, \mathrm{OR}=0.76,95 \% \mathrm{Cl}$ OR $[0.38,1.49]$.

Within rural scenes, $8.4 \%$ of all trials involved looked-but-failed-to-see error. Again, probability of looked-but-failed-to-see errors was not significantly different between SR ( $M$ $=9 \%, S E=2 \%)$ and NS $(M=7 \%, S E=1 \%)$ conditions, $\chi^{2}(1)=0.481, p=.488, B=0.20, S E=$ $0.29, \mathrm{OR}=1.22,95 \% \mathrm{Cl}$ OR $[0.69,2.17]$.

For the analysis directly comparing urban and rural looked-but-failed-to-see errors, including only trials where the change involved a car, motorcycle, or road sign, there was no significant main effect of sleep, $\chi^{2}(1)=0.965, p=.326, B=0.61, S E=0.26, \mathrm{OR}=1.84,95 \% \mathrm{Cl}$ OR $[0.54,6.24]$, and the sleep/environment interaction was not significant, $\chi^{2}(1)=2.581, p$ $=.108, B=-1.37, S E=0.85$. However, there was a significant effect of scene, $\chi^{2}(1)=8.095, p$ $=.004, B=1.50, S E=0.53, \mathrm{OR}=4.47,95 \% \mathrm{Cl}$ OR $[1.59,12.53]$. Observers were more likely to make looked-but-failed-to-see errors in urban $(M=8 \%, S E=1 \%)$ compared with rural $(M=$ $4 \%, S E=1 \%)$ scenes. This reflects the fact that many looked-but-failed-to-see errors in the rural scenes involved tree changes, which were not included in this analysis. 


\subsubsection{Time to first fixation and dwell time}

Time to first fixation on the target indicates the relative difficulty to visually locate the target, whereas dwell time indicates the relative difficulty of identifying a target once it has been fixated. Longer dwell times indicate that the participant required more time to cognitively process the target. These analyses included only trials in which the target was correctly identified and fixated. ${ }^{1}$

For the initial analyses considering urban and rural scenes separately, and including all correct trials regardless of change target type, there was no statistically significant effect of sleep condition time to first fixation or dwell time, as shown in Table 5. However, there was a nonsignificant trend whereby dwell time to identify targets was longer after sleep loss.

\section{Table 5}

Effects of sleep restriction on mean time to first fixation and dwell time in urban and rural scenes, with standard error shown in parentheses.

\begin{tabular}{|c|c|c|c|}
\hline & $\begin{array}{l}\text { Normal } \\
\text { Sleep }\end{array}$ & $\begin{array}{c}\text { Sleep } \\
\text { Restriction }\end{array}$ & Comparison \\
\hline \multicolumn{4}{|l|}{ Urban scenes } \\
\hline Time to first fixation (ms) & $1526(60)$ & $1626(99)$ & $\begin{array}{c}t(18)=1.033, p=.315 \\
d=1.18\end{array}$ \\
\hline Dwell time (ms) & $634(45)$ & $729(68)$ & $\begin{array}{c}t(18)=1.504, p=.150 \\
d=1.96\end{array}$ \\
\hline \multicolumn{4}{|l|}{ Rural scenes } \\
\hline Time to first fixation (ms) & $1586(76)$ & $1558(85)$ & $\begin{array}{c}t(19)=0.243, p=.811, \\
d=1.34\end{array}$ \\
\hline Dwell time (ms) & $640(56)$ & $758(97)$ & $\begin{array}{c}t(19)=1.512, p=.147 \\
d=1.28\end{array}$ \\
\hline
\end{tabular}

\subsubsection{Urban vs Rural comparison}

To directly compare dwell time and time to first fixation between urban and rural scenes, only trials with changes involving cars, motorcycles or road signs were included. Results are shown in Table 6. There were no statistically significant effects of sleep or environment, and

\footnotetext{
${ }^{1}$ Due to this criterion, one participant was excluded from the urban scene analyses, as they only fixated and identified one target out of 20) in the urban/SR condition and therefore had insufficient data to compute mean values.
} 
no interactions, although as with the $t$-test analyses reported above the main effect of sleep loss on dwell time approached significance.

\subsubsection{Dwell time on semantic interest areas}

RM-ANOVA was used to compare dwell times across five semantic interest areas (IAs; road, off-road left, off-road right, horizon, sky) with sleep (NS, SR) and environment (urban, rural) as additional within-subjects factors. Greenhouse-Geisser corrections were applied where Mauchly's test indicated significant violations of sphericity. There was no significant main effect of sleep condition, $F(1,19)=1.938, p=.180, \eta_{\mathrm{p}}^{2}=.09$, and none of the interactions involving sleep were statistically significant [sleep/environment: $F(1,19)=0.681, p=.420, \eta_{\mathrm{p}}^{2}$ $=.04 ;$ sleep/IA: $F(1.8,34.5)=2.099, p=.142, \eta_{\mathrm{p}}^{2}=.09 ;$ sleep/environment $/ \mathrm{IA}: F(1.8,34.0)=$ $\left.0.265, p=.744, \eta_{\mathrm{p}}^{2}=.01\right]$.

There were significant main effects of both environment, $F(1,19)=66.008, p<.001, \eta_{\mathrm{p}}^{2}=.78$ and IA $F(1.7,31.9)=49.24, p<.001, \eta_{\mathrm{p}}^{2}=.72$, and a significant environment/IA interaction, $F(1.7,31.5)=7.051, p=.005, \eta_{\mathrm{p}}^{2}=.27$. As shown in Figure 4, participants spent a greater proportion of time looking at the road in rural scenes, and spent a longer looking at the sky in urban scenes.
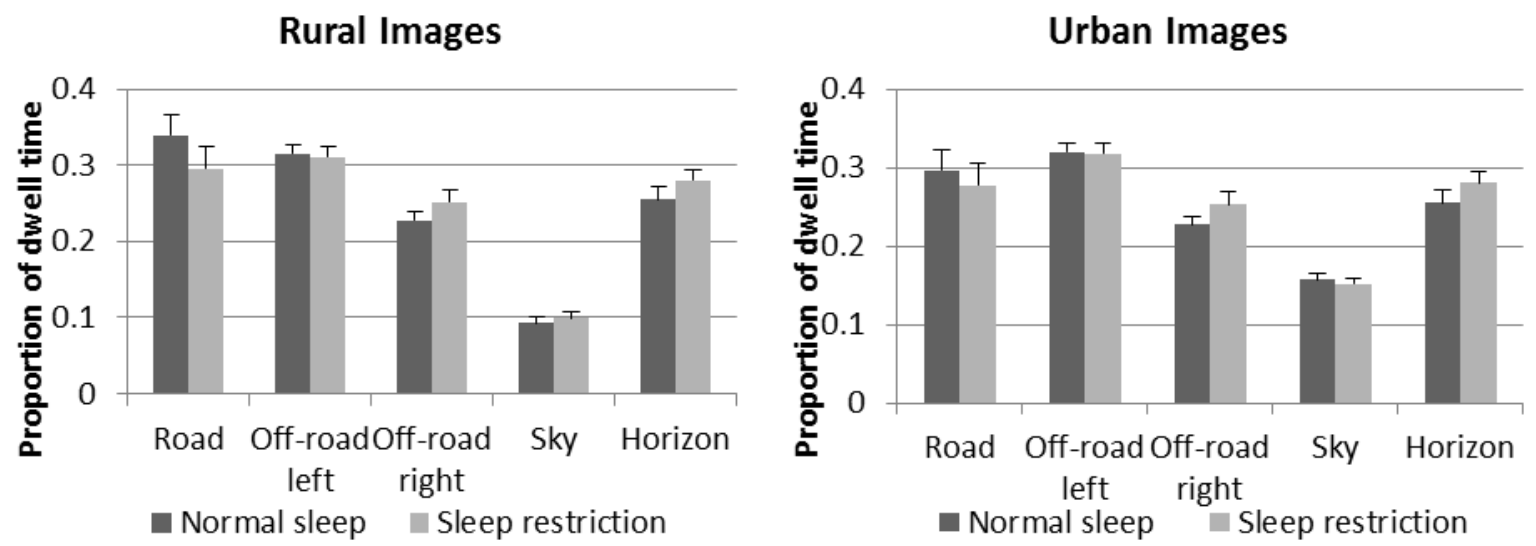

Fig. 4. Dwell time in areas of the road for change-absent images in rural (left) and urban (right) environments by sleep condition. Error bars represent standard error. 
Table 6

Effects of sleep restriction and environment on time to first fixation and dwell time in change-present rural and urban images (car, motorcycle and road sign changes only), standard error shown in parentheses.

\begin{tabular}{|c|c|c|c|c|c|c|c|}
\hline \multirow[b]{2}{*}{ Measure } & \multicolumn{2}{|c|}{ Normal Sleep } & \multicolumn{2}{|c|}{ Sleep Restriction } & \multicolumn{3}{|c|}{ Comparison } \\
\hline & Urban & Rural & Urban & Rural & Sleep & Environment & Interaction \\
\hline Dwell time (ms) & $663(53)$ & $659(52)$ & $741(72)$ & $787(97)$ & $\begin{array}{c}F=3.235, p \\
=.089, \eta_{\mathrm{p}}^{2}=.15\end{array}$ & $\begin{array}{c}F=0.271, p \\
=.609, \eta_{\mathrm{p}}^{2}=.02\end{array}$ & $\begin{aligned} & F=0.261, p \\
= & 616, \eta_{\mathrm{p}}^{2}=.01\end{aligned}$ \\
\hline Time to first fixation (ms) & $1397(41)$ & $1410(97)$ & $1587(114)$ & $1384(77)$ & $\begin{array}{c}F=0.741, p \\
=.401, \eta_{\mathrm{p}}^{2}=.04\end{array}$ & $\begin{array}{c}F=1.804, p \\
=.196, \eta_{\mathrm{p}}^{2}=.09\end{array}$ & $\begin{array}{c}F=1.743, p \\
=.203, \eta_{\mathrm{p}}^{2}=.09\end{array}$ \\
\hline
\end{tabular}

Note. Degrees of freedom were $(1,18)$ for all $F$ tests. 


\section{Discussion}

The aim of the current study was to examine the impact of mild sleep loss on change detection in urban and rural driving scenes. All participants were regular 7-8 hour sleepers who drove frequently. The mild sleep restriction (5 hours sleep) resulted in participants feeling significantly sleepier but did not significantly affect ocular indicators of sleepiness. The short testing paradigm employed did not induce time-on-task fatigue. Despite feeling sleepier, the results indicated that sleep loss does not significantly increase change blindness, nor does it systematically influence visual scanning patterns. There is some limited evidence that sleep loss may increase change detection RT in more visually complex urban driving scenes but not rural driving scenes. This finding may have implications for sleep-related crashes in urban environments. However, the finding of the urban/rural comparison was not supported by independent analysis of each environment, so should be considered with caution.

\subsection{Effect of sleep loss on eye movements and change detection accuracy}

Participants were equally able to maintain accuracy for detecting changes when alert and sleepy, in both urban and rural scenes. Maintained accuracy could be explained by visual scanning strategies as participants' eye movement patterns, including probability of fixating on the target, were not systematically affected by sleep loss. It was hypothesised that visual scanning strategies would be impaired by sleep loss, reflecting previous studies that have found visual search strategies increase in randomness following sleep loss (De Gennaro et al., 2001; Fransson et al., 2008; Glass et al., 2011). However, the level of sleep loss experienced by participants in the current study was very minor in comparison with the total sleep deprivation in previous research (De Gennaro et al., 2001; Fransson et al., 2008; Glass et al., 2011). This suggests that, among frequent drivers, visual scanning strategies are robust under minor sleep restriction. Further research is needed to confirm this finding under more extreme sleep loss.

Two reported outcomes of sleepiness are that drivers "tunnel" their vision spending a greater amount of gaze time looking at the centre of the road (Fors, Ahlström, \& Anund, 
2013), and that pupil size can be used to predict sleep related performance impairment (McClelland, Pilcher, \& Moore, 2010). If visual tunnelling were to occur it would be expected that a greater proportion of gaze time would occur within a smaller location. However, in the current study fixations at the 50th, 70th and 90th percentile were similar regardless of sleep condition, suggesting that visual tunnelling did not occur. Similarly, there was no significant difference in pupil size between sleep conditions. Together these findings indicate that the magnitude of sleepiness participants experienced was not sufficient to induce ocular indicators of sleepiness. It is possible that the maintenance of oculomotor control facilitated the maintained visual search approach, which helped maintain accurate change detection performance. Change detection impairment may have occurred if sleepiness were great enough to have induced ocular indicators of sleepiness.

\subsection{Effects of sleep loss on change detection response time}

In general, change detection RTs were not slowed by sleep loss. The two exceptions were interactions between sleep condition and environment for the slowest $10 \%$ RTs and RT variability. When sleepy the slowest $10 \%$ RTs became slower for change-present urban images but became faster for rural images. Opposing effects were also found for RT variability, with variability increasing for urban images but decreasing for rural following sleep loss. However, it should be noted that the effects of sleep loss on RT emerged in the urban/rural comparison, which only analysed changes involving cars, motorcycles and signs, whereas in the separate analyses including all target types (i.e., pedestrians and traffic lights in urban scenes; animals and trees in rural scenes) there was no effect of sleep loss on RT. It is possible that the significant finding may be an artefact of external stimuli factors. More research is needed to confirm whether this is a robust finding and to understand why sleep loss might enhance the detection of changes in rural environments but impair it in urban environments. One potential influencing factor is the amount of visual complexity within an image. Urban images contain a greater number of elements to be inspected in order to identify a change. For alert participants change detection RT is quicker with rural images compared to rural images (Beanland et al., 2017). It is possible that the added visual complexity interacts with sleep loss to further impair performance when attempting to 
detect changes in urban images. Further research is needed to fully understand the impact of visual complexity on change blindness.

If the impact of sleep loss on urban change detection RT was robust this could mean that slowed change detection contributes towards sleep-related crashes in urban (but perhaps not rural) environments. While there are many similarities between sleep-related crashes on urban and rural roads, one difference is that drivers in urban sleep-related crashes are more likely to be carrying passengers than those on rural roads (Filtness et al., 2017). The presence of passengers and a possible susceptibility to slowed change detection could combine to contribute towards crashes. Sleep loss increases drivers' propensity to be distracted (Anderson \& Horne, 2013) and change blindness is more common when engaging in a secondary task such as talking (McCarley et al., 2004). The current study was conducted under quiet controlled conditions; it is possible that the interaction between sleep loss and driving environment may have been greater had an additional distractor been present.

It is well established that sleep loss impairs RT to the PVT, a sustained vigilance task, e.g. (Anderson et al., 2010; Van Dongen et al., 2003). However, the current study demonstrated that sleep loss does not have a similar effect on change detection accuracy or mean RT to identify changes. This disparity may result from fundamental differences in the nature of the tasks. Specifically, the PVT is a very simple response task, in which participants react to intermittent stimuli. Change detection tasks are much more complex, involving not only visual attention but also working memory. The PVT stimuli are displayed at random intervals requiring sustained vigilance at all times, conversely change-blindness paradigms alternate between images and a blank screen. Different images are used for each change detection trial. It may be that the flickering between images is stimulating enough to maintain attentional focus despite feeling sleepy. Furthermore, the alternating images may cue participants to focus their attention, and allow them to briefly "zone out" during the known periods of no stimuli (blanks). This on/off attentional focus may have negated the eyes open lapses which can be observed using the PVT (Anderson et al., 2010). 


\subsection{Future directions}

The biggest strength of the current study is the controlled experimental approach. However, the extent to which these lab based findings generalise to real-world driving is unclear. In the current study participants were instructed to look for changes in each image pair. In reality drivers are not cued to the potential presence of a change at a specifiv time, but rather must continually engage in searching for changes. Given that physically driving requires detection of dynamic changes while under the added task load of maintaining vehicle control. Previous research has demonstrated change blindness to be greater with dynamic compared to static stimuli (Velichkovsky et al., 2002). This suggests that change blindness may be more likely to occur when driving compared with observing static images. Therefore, the impact of sleep loss should be further investigated in a dynamic environment, including some change detection tests where participants are not explicitly cued to look for changes.

Another strength of the current study is that it was designed to replicate the magnitude of sleepiness which might be experienced in everyday driving. For this reason total sleep deprivation protocol was avoided. Although the results of this study may be considered to have greater real world validity than if a total sleep deprivation protocol had been used, it is possible that a greater degree of sleepiness may have influenced change blindness.

It should be noted that participants in the current study drove frequently and had held a driving licence for at least 18 months. Previous research has demonstrated that experienced drivers are better able to adapt their visual scanning patterns to meet situational demands (e.g. Falkmer \& Gregersen, 2005; Underwood, 2007). It is probable that if participants were novice drivers they may not have utilised the same visual scanning patterns. Younger drivers are known to exhibit greater driving impairment in response to sleep loss than older drivers (Filtness et al., 2012), therefore it is possible that the visual scanning patterns (and subsequently change detection performance) of younger novice drivers may be influenced by sleep loss. However, the impact of experience is also influenced by instructions given to participants (e.g. in observing the image consider if it is safe to cross the intersection) Previous research has demonstrated that when given driving manoeuvre based instructions, experienced drivers have a selective search strategy to viewing driving scenes. Changes to 
objects that would not be impacted by the considered manoeuvre are therefore more likely to be noticed by novice drivers (Koustanaï et al., 2012). It is important for future research to consider novice drivers because young drivers are consistently over represented in sleeprelated crashes (Filtness et al., 2017; Horne \& Reyner, 1995; Philip et al., 2014). Differences between experienced and novice drivers are often observed in scientific research, therefore it is suggested that future research focus on these two groups. However, it is also possible that varying driving exposure within experienced drivers may influence results. In the current study weekly driving exposure ranged from 1-42 hours. Future research may wish to consider the impact of driving regularity on change blindness.

\section{Conclusion}

Overall, it appears that the impact of sleep loss on change detection is at most small and subtle, with most analyses not indicating any statistically significant differences. The type of visual scene had greater influences on ability to detect changes with participants being better at detecting changes in rural scenes compared with urban scenes. However, as with any "null finding", the lack of significance should be interpreted with caution. Specifically, the lack of a statistically significant difference does not mean that two conditions are the same.

Nonetheless, both driver sleepiness (Åkerstedt, 2000) and driver attention failures (including failure to detect changes Beanland et al., 2013), are significant road safety problems associated with increased crash risk. Future research is vital to understand what factors increase the risk of change blindness for drivers in order for appropriate countermeasures to be developed.

\section{Acknowledgements}

This work was supported by an NRMA-ACT Road Safety Trust Grant. Vanessa Beanland is supported by an Australian Research Council Discovery Early Career Researcher Award [grant DE150100083]. We thank Rhiannon Jeans for experiment programming and 
assistance with data collection, Alexander Smith for assistance with data collection, and Shannon Webb and Erin Walsh for assistance with preparing the photographic stimuli.

\section{References}

Åkerstedt, T. (2000). Consensus statement: Fatigue and accidents in transport operations. Journal of Sleep Research, 9(4), 395-395.

Åkerstedt, T., \& Gillberg, M. (1990). Subjective and objective sleepiness in the active individual. International Journal of Neuroscience, 52(1-2), 29-37.

Anderson, C., \& Horne, J. A. (2013). Driving drowsy also worsens driver distraction. Sleep Medicine, 14(5), 466-468.

Anderson, C., Wales, A. W., \& Horne, J. A. (2010). PVT lapses differ according to eyes open, closed, or looking away. Sleep, 33(2), 197-204.

Anund, A., Kecklund, G., Vadeby, A., Hjälmdahl, M., \& Åkerstedt, T. (2008). The alerting effect of hitting a rumble strip-A simulator study with sleepy drivers. Accident Analysis \& Prevention, 40(6), 1970-1976.

Armstrong, K., Filtness, A. J., Watling, C. N., Barraclough, P., \& Haworth, N. (2013). Efficacy of proxy definitions for identification of fatigue/sleep-related crashes: An Australian evaluation. Transportation Research Part F: Traffic Psychology and Behaviour, 21, 242-252.

Basner, M., \& Dinges, D. F. (2011). Maximizing sensitivity of the psychomotor vigilance test (PVT) to sleep loss. Sleep, 34(5), 581-591.

Basner, M., Rubinstein, J., Fomberstein, K. M., Coble, M. C., Ecker, A., Avinash, D., et al. (2008). Effects of night work, sleep loss and time on task on simulated threat detection performance. Sleep, 31(9), 1251-1259.

Batchelder, S., Rizzo, M., Vanderleest, R., \& Vecera, S. (2003). Traffic scene related change blindness in older drivers. Paper presented at the Proceedings of the 2nd International Driving Symposium on Human Factors in Driver Assessment, Training, and Vehicle Design, pp. 177-181.

Beanland, V., Filtness, A. J., \& Jeans, R. (2017). Change detection in urban and rural driving scenes: Effects of target type and safety relevance on change blindness. Accident Analysis \& Prevention, 100, 111-122.

Beanland, V., Fitzharris, M., Young, K. L., \& Lenné, M. G. (2013). Driver inattention and driver distraction in serious casualty crashes: Data from the Australian national crash in-depth study. Accident Analysis \& Prevention, 54, 99-107. 
Caird, J. K., Edwards, C. J., Creaser, J. I., \& Horrey, W. J. (2005). Older driver failures of attention at intersections: Using change blindness methods to assess turn decision accuracy. Human Factors, 47(2), 235-249.

Cajochen, C., Khalsa, S. B., Wyatt, J. K., Czeisler, C. A., \& Dijk, D. J. (1999). EEG and ocular correlates of circadian melatonin phase and human performance decrements during sleep loss. The American Journal of Physiology, 277(3 Pt 2), R640-9.

Charlton, S. G., \& Starkey, N. J. (2013). Driving on familiar roads: Automaticity and inattention blindness. Transportation Research Part F: Traffic Psychology and Behaviour, 19, 121-133.

Clark, B., \& Warren, N. (1939). The effect of loss of sleep on visual tests. American Journal of Optometry, 16, 80-95

Connor, J., Norton, R., Ameratunga, S., Robinson, E., Civil, I., Dunn, R., et al. (2002). Driver sleepiness and risk of serious injury to car occupants: Population based case control study. BMJ (Clinical Research Ed.), 324(7346), 1125.

De Gennaro, L., Ferrara, M., Curcio, G., \& Bertini, M. (2001). Visual search performance across $40 \mathrm{~h}$ of continuous wakefulness: Measures of speed and accuracy and relation with oculomotor performance. Physiology \& Behavior, 74(1), 197-204.

De Gennaro, L., Ferrara, M., Urbani, L., \& Bertini, M. (2000). Oculomotor impairment after 1 night of total sleep deprivation: A dissociation between measures of speed and accuracy. Clinical Neurophysiology, 111(10), 1771-1778.

Dinges D.F., \& Kribbs N.B. (1991) Performing while sleepy: effects of experimentally-induced sleepiness. In: Monk TH, ed. Sleep, sleepiness and performance. Chichester, U.K.: Wiley, 1991:97-128

Dinges, D. F., Pack, F., Williams, K., Gillen, K. A., Powell, J. W., Ott, G. E., et al. (1997). Cumulative sleepiness, mood disturbance and psychomotor vigilance performance decrements during aweek of sleep restricted to 4-5 hours per night. Sleep, 20, 267-277.

Falkmer, T., \& Gregersen, N. P. (2005). A comparison of eye movement behavior of inexperienced and experienced drivers in real traffic environments. Optometry \& Vision Science, 82(8), 732-739.

Filtness, A. J., Armstrong, K. A., Watson, A., \& Smith, S. S. (2017). Sleep-related vehicle crashes on low speed roads. Accident Analysis \& Prevention, 99, 279-286.

Filtness, A. J., Reyner, L. A., \& Horne, J. A. (2012). Driver sleepiness-comparisons between young and older men during a monotonous afternoon simulated drive. Biological Psychology, 89(3), 580-583. 
Fors, C., Ahlström, C., \& Anund, A. (2013). Simulator validation with respect to driver sleepiness and subjective experiences: Final report of the project SleepEYE II, part 1 Retrieved from: http://www.diva-portal.org/smash/get/diva2:674216/FULLTEXT01.pdf

Fransson, P., Patel, M., Magnusson, M., Berg, S., Almbladh, P., \& Gomez, S. (2008). Effects of 24-hour and 36-hour sleep deprivation on smooth pursuit and saccadic eye movements. Journal of Vestibular Research, 18(4), 209-222.

Galpin, A., Underwood, G., \& Crundall, D. (2009). Change blindness in driving scenes. Transportation Research Part F: Traffic Psychology and Behaviour, 12(2), 179-185.

Glass, B. D., Maddox, W. T., Bowen, C., Savarie, Z. R., Matthews, M. D., Markman, A. B., et al. (2011). The effects of 24-hour sleep deprivation on the exploration-exploitation trade-off. Biological Rhythm Research, 42(2), 99-110.

Graw, P., Kräuchi, K., Knoblauch, V., Wirz-Justice, A., \& Cajochen, C. (2004). Circadian and wake-dependent modulation of fastest and slowest reaction times during the psychomotor vigilance task. Physiology \& Behavior, 80(5), 695-701.

Harrison, Y., \& Horne, J. (1998). Sleep loss impairs short and novel language tasks having a prefrontal focus. Journal of Sleep Research, 7(2), 95-100.

Harrison, Y., Horne, J. A., \& Rothwell, A. (2000). Prefrontal neuropsychological effects of sleep deprivation in young adults--a model for healthy aging? Sleep, 23(8), 1067-1073.

Helton, W. S., Weil, L., Middlemiss, A., \& Sawers, A. (2010). Global interference and spatial uncertainty in the sustained attention to response task (SART). Consciousness and Cognition, 19(1), 77-85.

Horne, J.A. (1975). Binocular convergence in man during total sleep deprivation. Biological Psychology, 3(4), 309-319.

Horne, J. A., \& Reyner, L. A. (1995). Sleep related vehicle accidents. BMJ, 310(6979), 565-567.

Johns, M. W. (1991). A new method for measuring daytime sleepiness: The Epworth Sleepiness Scale. Sleep, 14(6), 540-545.

Koustanaï, A., Van Elslande, P., \& Bastien, C. (2012). Use of change blindness to measure different abilities to detect relevant changes in natural driving scenes. Transportation Research Part F: Traffic Psychology and Behaviour, 15(3), 233-242.

Liang, K., \& Zeger, S. L. (1986). Longitudinal data analysis using generalized linear models. Biometrika, 13-22.

Marchetti, L. M., Biello, S. M., Broomfield, N. M., MACMAHON, K., \& Espie, C. A. (2006). Who is pre-occupied with sleep? A comparison of attention bias in people with psychophysiological insomnia, delayed sleep phase syndrome and good sleepers using the induced change blindness paradigm. Journal of Sleep Research, 15(2), 212-221. 
Martens, M. H., \& Fox, M. R. (2007). Do familiarity and expectations change perception? drivers' glances and response to changes. Transportation Research Part F: Traffic Psychology and Behaviour, 10(6), 476-492.

McCarley, J. S., Vais, M. J., Pringle, H., Kramer, A. F., Irwin, D. E., \& Strayer, D. L. (2004). Conversation disrupts change detection in complex traffic scenes. Human Factors, 46(3), 424-436.

McClelland, L. E., Pilcher, J. J., \& Moore, D. D. (2010). Oculomotor measures as predictors of performance during sleep deprivation. Aviation, Space, and Environmental Medicine, 81(9), 833-842.

Mouloua, M., \& Parasuraman, R. (1995). Aging and cognitive vigilance: Effects of spatial uncertainty and event rate. Experimental Aging Research, 21(1), 17-32.

Nilsson, J. P., Söderström, M., Karlsson, A. U., Lekander, M., Åkerstedt, T., Lindroth, N. E., et al. (2005). Less effective executive functioning after one night's sleep deprivation. Journal of Sleep Research, 14(1), 1-6.

Philip, P., Chaufton, C., Orriols, L., Lagarde, E., Amoros, E., Laumon, B., et al. (2014). Complaints of poor sleep and risk of traffic accidents: A population-based case-control study. PloS One, 9(12), e114102.

Rensink, R. A., O'Regan, J. K., \& Clark, J. J. (1997). To see or not to see: The need for attention to perceive changes in scenes. Psychological Science, 8(5), 368-373.

Rizzo, M., Sparks, J., McEvoy, S., Viamonte, S., Kellison, I., \& Vecera, S. P. (2009). Change blindness, aging, and cognition. Journal of Clinical and Experimental Neuropsychology, 31(2), 245-256.

Sänger, J., \& Wascher, E. (2011). The influence of extrinsic motivation on competition-based selection. Behavioural Brain Research, 224(1), 58-64.

Theeuwes, J. (1994). Stimulus-driven capture and attentional set: Selective search for color and visual abrupt onsets. Journal of Experimental Psychology: Human Perception and Performance, 20(4), 799-806.

Underwood, G. (2007). Visual attention and the transition from novice to advanced driver. Ergonomics, 50(8), 1235-1249.

Van Dongen, H. P., \& Dinges, D. F. (2000). Circadian rhythms in fatigue, alertness, and performance. Principles and Practice of Sleep Medicine, 3, 391-399.

Van Dongen, H. P., Maislin, G., Mullington, J. M., \& Dinges, D. F. (2003). The cumulative cost of additional wakefulness: Dose-response effects on neurobehavioral functions and sleep physiology from chronic sleep restriction and total sleep deprivation. Sleep, 26(2), 117-129. 
Velichkovsky, B. M., Dornhoefer, S. M., Kopf, M., Helmert, J., \& Joos, M. (2002). Change detection and occlusion modes in road-traffic scenarios. Transportation Research Part F: Traffic Psychology and Behaviour, 5(2), 99-109.

Werner, S., \& Thies, B. (2000). Is" change blindness" attenuated by domain-specific expertise? an expert-novices comparison of change detection in football images. Visual Cognition, 7(1-3), 163-173. 